\title{
DECOMPOSITIONS OF DIFFERENTIABLE SEMIGROUPS
}

\author{
JOHN P. HOLMES
}

(Communicated by Jonathan M. Rosenberg)

\begin{abstract}
A differentiable semigroup is a topological semigroup $(S, *)$ in which $S$ is a differentiable manifold based on a Banach space and the associative multiplication function $*$ is continuously differentiable. If $e$ is an idempotent element of such a semigroup we show that there is an open set $U$ containing $e$ so that there is a $C^{1}$ retraction $\Phi$ of $U$ into the set of idempotents of $S$ so that $\Phi(x) \Phi(y)=\Phi(x)$ for $x$ and $y$ in $U$ and $x \Phi(x)$ is in the maximal subgroup of $S$ determined by $\Phi(x)$ for each $x$ in $U$. This leads to a natural decomposition of $S$ near $e$ into the union of a collection of mutually disjoint and mutually homeomorphic local differentiable subsemigroups whose intersections with $U$ are the point inverses under $\Phi$. In case $S$ is the semigroup under composition of continuous linear transformations on a Banach space, in the case of a nontrivial idempotent $e$, the existence of $\Phi$ implies that operators near an $e$ have nontrivial invariant subspaces. A dual right handed result holds.
\end{abstract}

\section{INTRODUCTION}

If $e$ is an idempotent element of the semigroup $(S, *)$, denote by $H(e)$ the largest subgroup of $S$ containing $e$. The following sets are subsemigroups determined by $e$ :

$$
\begin{aligned}
S_{e} & =\{x: \quad x e \text { is in } H(e)\}, \\
e^{S} & =\{x: e x \text { is in } H(e)\} .
\end{aligned}
$$

Each of these has $H(e)$ as a homomorphic image. For instance the function which sends $x$ to $x e$ is a homomorphism from $S_{e}$ onto $H(e)$. In addition $H(e)$ is a minimal left ideal in $S_{e}$ and $H(e)$ is a minimal right ideal in $e_{e}$. Our main purpose here is to show that the $S_{e}$ subsemigroups are a basic building block of differentiable semigroups in the sense that, in such a semigroup, a neighborhood of each idempotent has a topological product structure in which each factor is a $C^{1}$ submanifold, one factor consists of idempotents $e$, and the

Received by the editors July 31, 1989 and, in revised form, October 24, 1989; presented to the Conference on Topologishe und Analytische Halbgruppen at the Mathematische Forshungs Institute-Oberwolfach, February 1989.

1980 Mathematics Subject Classification (1985 Revision). Primary 22A15, 58B25; Secondary 47A 15.

Key words and phrases. $C^{1}$ semigroup, idempotent, retraction, invariant subspace. 
fiber over $e$ is homeomorphic with a neighborhood of $e$ in $S_{e}$. We show this by constructing a differentiable retraction $\Phi$ on a neighborhood of $e$ whose point inverses are the intersection of the $S_{e}$ 's with the neighborhood. A similar statement holds for the ${ }_{e} S$ 's.

One way to construct examples of differentiable semigroups is the following. Suppose each of $A$ and $B$ is a differentiable manifold, $G$ is a Lie group (or, more generally, a differentiable semigroup), and $f$ is a $C^{1}$ map from $B \times$ $A$ into $G$. Let $S=A \times G \times B$ and define $*$ by $(a, g, b) *(c, h, d)=$ $(a, g f(b, c) h, d)$. The result is a differentiable semigroup $(S, *)$ called a Rees product semigroup or, in case $G$ is a group, a paragroup. It follows from Theorem 1 below that, for general differentiable semigroups, if $e$ is not isolated in the set of idempotents then $e$ is contained in a nontrivial sub-paragroup. If $e$ is not central this implies that the decomposition described above, is nontrivial in the sense that the factor in the products mentioned above, which consists of idempotents, is not a singleton.

Another example results from taking $S$ to be an associative Banach algebra and $*$ to be the algebra multiplication. For instance, $S$ could be the algebra of continuous linear transformations of a Banach space $X$ with the composition multiplication. Our main results, Theorems 5 and 7, appear to be new even in this setting.

\section{BACKGROUND}

This is a continuation of the study of semigroups with differentiable multiplication begun in [5-7]. These papers describe their structure near an idempotent element $e$.

The semigroup $(S, *)$ is differentiable provided that $S$ is, in addition to being a topological semigroup, a differentiable manifold based upon some Banach space $X$, and the associative multiplication function $(x, y) \rightarrow x y$ is $C^{1}$.

First we state results from [5-8] which will be used in the sequel. The fundamental analytical tool is the following theorem. A preliminary version of it may be found in [5] and the final form in [8].

Theorem 1. Suppose $S$ is a $C^{1}$ manifold and $f$ is a $C^{1}$ retraction $(f f=f)$ from an open set of $S$ into $S$. Each component of the image of $f$ is a $C^{1}$ submanifold of $S$ and, for each $x$ in the image of $f$, there is an open set $U$ containing $x$ and $a C^{1}$ retraction $g$ with domain $U$ and image the intersection of $U$ with $f^{-1}(\{x\})$. Moreover, $U$ can be chosen so that the map

$$
u \rightarrow(f(u), g(u))
$$

is a $C^{1}$ homeomorphism of $U$ onto $f(U) \times g(U)$.

If $e$ is in $E(S)$, the set of idempotent elements of $S$, let $P$ be defined on $S$ by $P(x)=e x e$. By Theorem 1 , the image $e S e$ of $P$ is a local $C^{1}$ semigroup with identity element. That such an object is a local Lie group has 
been observed by many, for example Birkhoff [2]. These two observations form the basis of the following theorem from [5].

Theorem 2. If $e$ is in $E(S)$ then the maximal subgroup $H(e)$, of $S$ which contains $e$, is a Lie group and is an open subset of the image of the $C^{1}$ retraction $P$.

Suppose $e$ is an idempotent in the differentiable semigroup $(S, *)$. From Theorem 2 we know that $S^{e}=P^{-1}(H(e))$ is open. In the following let $z^{-1}$ denote the inverse of $z$ in $H(e)$. If we set

$$
\begin{aligned}
& L(x)=x P(x)^{-1} \\
& R(x)=P(x)^{-1} x,
\end{aligned}
$$

then $L$ and $R$ are $C^{1}$ retractions of the open set $S^{e}$. The next theorem summarizes results from [6 and 7] concerning $L$ and $R$. The topological parts of the conclusion follow from Theorems 1 and 2. The rest of the conclusions have algebraic arguments.

Theorem 3. The following statements hold for $L$ and $R$.

(a) Each of $L$ and $R$ is a $C^{1}$ retraction on $S^{e}$.

(b) If each of $x$ and $y$ is in $S^{e}$ then $L(x) L(y)=L(x)$ and $R(x) R(y)=$ $R(y)$. So $\operatorname{Im}(L)$ is a differentiable left-zero-subsemigroup, and $\operatorname{Im}(R)$ is a differentiable right-zero-subsemigroup.

(c) If each of $l$ and $l^{\prime}$ is in $\operatorname{Im}(L)$ then $l H\left(l^{\prime}\right)=H(l)$. The function $g \rightarrow l g$ is a $C^{1}$-isomorphism from $H\left(l^{\prime}\right)$ onto $H(l)$.

(d) If each of $r$ and $r^{\prime}$ is in $\operatorname{Im}(R)$, then $H\left(r^{\prime}\right) r=H(r)$. The function $g \rightarrow g r$ is a $C^{1}$-isomorphism from $H\left(r^{\prime}\right)$ to $H(r)$.

(e) The sets $\operatorname{Im}(L) H(e)$ and $H(e) \operatorname{Im}(R)$ are differentiable subsemigroups of $S$ which are $C^{1}$ isomorphic with the product semigroups $\operatorname{Im}(L) \times$ $H(e)$ and $H(e) \times \operatorname{Im}(R)$, respectively. The functions $(l, g) \rightarrow \lg$ and $(g, r) \rightarrow g r$, respectively, are $C^{1}$-isomorphisms.

(f) The set $\mathrm{Se} \cap S^{e}$ is equal to $\operatorname{Im}(L) H(e)$. The set $e S \cap S^{e}$ is equal to $H(e) \operatorname{Im}(R)$.

\section{MAIN RESUlts}

We now will show that $\operatorname{Im}(L) H(e)$ and $H(e) \operatorname{Im}(R)$ are the images of $C^{1}$ retractions whose domains are open sets of $S$. The retraction onto $\operatorname{Im}(L) H(e)$ maps that portion of the subsemigroup $S_{t}$ which is near the image of the retraction onto $H(t)$, for some idempotent $t$ near $e$. This retraction in turn is used to construct another retraction onto a subset of the idempotents near $e$ so that the point inverse of an idempotent $t$ in its image is the part of $S_{t}$ near $e$. The results claimed in the introduction follow from applying Theorem 1 to this retraction. A similar statement holds for the retraction onto $H(e) \operatorname{Im}(R)$ and the ${ }_{t} S$ subsemigroups. 
From Theorem 3 it is apparent that $\operatorname{Im}(L) H(e)$ is an open subsemigroup of the left ideal and differentiable subsemigroup $S e$. Similarly $H(e) \operatorname{Im}(R)$ is an open subsemigroup of the right ideal $e S$. Theorem 4 shows that $\operatorname{Im}(L) H(e)$ and $H(e) \operatorname{Im}(R)$ are local left and right ideals, respectively. It is the algebraic preliminary needed for setting up an application of the implicit function theorem which will yield the retractions mentioned above.

Theorem 4. There is an open set $U$ of $S$ containing $e$ and open sets $A$ and $B$ of $\operatorname{Im}(L)$ and $\operatorname{Im}(R)$, respectively, containing $e$ so that if $x$ is in $U, y$ is in $A H(e)$, and $z$ is in $H(e) B$, then $x y$ is in $\operatorname{Im}(L) H(e)$ and $z x$ is in $H(e) \operatorname{Im}(R)$.

Proof. It is clear that $\operatorname{Im}(R) \operatorname{Im}(L)$ is contained in $\operatorname{Im}(P)$. So, since $H(e)$ is open in $\operatorname{Im}(P)$, one may use continuity of multiplication to choose open sets $A$ and $B$ of $\operatorname{Im}(L)$ and $\operatorname{Im}(R)$, respectively, containing $e$ so that $B A$ is contained in $H(e)$. From Theorem 3(e) we have that $V=A H(e)$ and $W=H(e) B$ are open in $\operatorname{Im}(L) H(e)$ and $H(e) \operatorname{Im}(R)$, respectively, and hence, by Theorem 3(f), are open in $S e$ and $e S$, respectively. Since $e^{2}=e$ is in each of $V$ and $W$, we may choose $U$ open in $S$ and containing $e$ so that if $x$ is in $U$ then $x e$ is in $V$ and $e x$ is in $W$.

Suppose that $x$ is in $U$ and $y$ is in $A H(e)$. Choose $l$ in $A$ and $g$ in $H(e)$ so that $y=l g$. Note that $x y$ is in Se since $g e=g$. But $e(x y) e=$ $e x(l g)=h r l g$, for some $h$ in $H(e)$ and $r$ in $B$, since $e x$ is in $W$. But $r l$ is in $H(e)$, so it follows that $x y$ is in $S e \cap S^{e}$. Hence, by Theorem 3(f), the element $x y$ is in $\operatorname{Im}(L) H(e)$. The remainder of the argument is similar.

Theorem 5. Suppose $(S, *)$ is a differentiable semigroup, $e$ is an idempotent of $S$, and $L$ is as in Theorem 3. There is an open set $O$ containing $\operatorname{Im}(L) H(e)$ so that there is a unique continuous function $G$ from $O$ onto $\operatorname{Im}(L) H(e)$ satisfying $x L(G(x))=G(x)$. For each $x$ in $\operatorname{dom}(G)$, we have that $G(x)$ is in $H(L(G(x)))$. The function $G$ is a $C^{1}$ retraction.

Proof. If $x$ is in $S^{e}$ then so is $x e$, since $e(x e) e=e x e$. Thus $\left(S^{e}\right) e$ is contained in $S e \cap S^{e}$. But, if $x$ is in $S e \cap S^{e}$ then $x=x e$ is in $\left(S^{e}\right) e$ so $\left(S^{e}\right) e=\operatorname{Se} \cap S^{e}$.

Suppose $S$ is a manifold based upon the Banach space $X$. By Theorem 1, the local ideal $\left(S^{e}\right) e$ is a differentiable submanifold of $S^{e}$ based upon some closed subspace $Y$ of $X$. Suppose $p$ is in $H(e)$, and choose local coordinates $f$ and $g$ at $p$ in $S^{e}$ and at $p$ in $\left(S^{e}\right) e$, respectively, which are compatible with the differentiable structure and map $p$ to 0 . Using Theorem 4 choose $U^{\prime}$ open in $S^{e}$, containing $p$ and contained in $\operatorname{dom}(f)$, and an open subset $A^{\prime}$ of $\operatorname{Im}(L)$ containing $e$ so that if $V^{\prime}=A^{\prime} H(e)$ then $U^{\prime} V^{\prime}$ is contained in $\left(S^{e}\right) e$. Note that $p$ is in $V^{\prime}$.

Note that $L(p)=p(e p e)^{-1}=e$, so $p L(p)=p$. Using continuity of multiplication and $L$, choose open subsets $U$ and $V$ of $U^{\prime}$ and $V^{\prime}$, respectively, containing $p$ so that $L(V)$ is contained in $A^{\prime}$ and $U L(V)$ is contained in $\operatorname{dom}(g)$. Define the open sets $C$ and $D$ of $\operatorname{Im}(f)$ and $\operatorname{Im}(g)$, 
respectively, by $C=f(U)$ and $D=g(V)$. Now, if $x$ is in $C$ and $y$ is in $D$, we have $f^{-1}(x)$ is in $U$ and $g^{-1}(y)$ is in $V$ so that $f^{-1}(x) L\left(g^{-1}(y)\right)$ is in $\operatorname{dom}(g)$. Thus we may define the function $F$ on $C \times D$ into $Y$ by $F(x, y)=y-g\left(f^{-1}(x) L\left(g^{-1}(y)\right)\right)$.

By choice of $f$ and $g$ we have $F$ continuously differentiable on $C \times D$. Note that, for each $z$ in $D$,

$$
F(0, z)=z-g\left(p L\left(g^{-1}(z)\right)=z-g(p)=z\right.
$$

since, by Theorem $3(b)$, we have

$$
p \operatorname{Im}(L)=p e \operatorname{Im}(L)=p\{e\}=\{p\} .
$$

It follows that $D_{2} F(0,0)=I$, where $I$ is the identity transformation on $Y$. Hence, by the implicit function theorem, see for instance [3], there are open sets $P$ and $Q$ of $X$ and $Y$, respectively, each containing 0 so that there is a unique continuous function $h$ from $P$ into $Q$ satisfying $h(0)=0$ and $F(x, h(x))=F(0,0)=0$ for each $x$ in $P$. Moreover, $h$ is continuously differentiable and $h^{\prime}(x)=-D_{2} F(x, h(x))^{-1} D_{1} F(x, h(x))$.

If $x$ is in the open set $f^{-1}(P)$ then, from the definitions of $F$ and $h$, we have $h(f(x))=g\left(x L\left(g^{-1}(h(f(x)))\right)\right)$. Thus, if $G(x)=g^{-1}(h(f(x)))$ we have that $G(x)$ is in $\operatorname{Im}(L) H(e)$ and $x L(G(x))=G(x)$. Moreover, since $h$ is $C^{1}$, then $G$ is $C^{1}$.

If $G(x)=l z$ for $l$ in $\operatorname{Im}(L)$ and $z$ in $H(e)$, then $L(G(x))=L(l z)=$ $l z(e l z e)^{-1}=l z z^{-1}=l e=l$. By Theorem $3(\mathrm{c}) l z$ is in $H(l)$, so it follows that $x L(G(x))=G(x)$ is in $H(L(G(x)))$.

To see that $G$ is a retraction consider applying the preceding argument to the $C^{1}$ semigroup $\operatorname{Im}(L) H(e)$. For $y$ in $\operatorname{Im}(L) H(e)$ there is an open set containing $y$ so that there is a unique continuous function $K$ satisfying $z L(K(z))=K(z)$ on the open set. If $x=l z$ for $l$ in $\operatorname{Im}(L)$ and $z$ in $H(e)$, then $l z$ is in $H(l)$ and, from the previous paragraph, $L(l z)=l$, so $\operatorname{lz} L(l z)=l z$. Thus, the identity function is one such function. But, the restriction of $G$ is another such function. So, on $(\operatorname{Im}(L) H(e)) \cap \operatorname{dom}(G)$, we have $G(x)=x$.

From this and the uniqueness of $h$ we see that there are neighborhoods $O_{1}$ and $O_{2}$ of $y$ in $S$ and $\operatorname{Im}(L) H(e)$, respectively, so that $G$ is the only continuous function defined from $O_{1}$ onto $O_{2}$ which satisfies $x L(G(x))=$ $G(x)$. But, if $l$ is in $\operatorname{Im}(L)$ and $x=l^{\prime} z$ is in $\operatorname{Im}(L) H(e)$ for $l^{\prime}$ in $\operatorname{Im}(L)$ and $z$ in $H(e)$, then

$$
\begin{aligned}
x(l x l)^{-1} & =x\left(l l^{\prime}(z e) l\right)^{-1} \\
& =x(l z)^{-1} \quad(\text { by Theorem 3(b)) } \\
& =x l z^{-1} \quad(\text { by Theorem 3(c)) } \\
& =(x e) l(e x e)^{-1}=L(x) \quad(\text { by Theorem 3(b)). }
\end{aligned}
$$


Thus the equation $x L(y)=y$ is independent of the idempotent $e$ for $y$ in $\operatorname{Im}(L) H(e)$.

It follows that this equation implicitly defines a unique continuous function on a neighborhood of $y$ for each $y$ in $\operatorname{Im}(L) H(e)$. Any pair of these functions must hence agree on the intersection of their domain of uniqueness. Thus we have the existence of an open set $O$ containing $\operatorname{Im}(L) H(e)$ so that there is a unique continuous function $G$ from $O$ into $\operatorname{Im}(L) H(e)$ satisfying $x L(G(x))=$ $G(x)$ for each $x$ in $O$. Moreover, we have seen that, for $x$ in $\operatorname{Im}(L) H(e)$, we have $G(x)=x$. Thus, $G G=G$ and we are done with a proof of Theorem 5.

Recall from the introduction that, for any idempotent $e$, we have defined the sets

$$
\begin{aligned}
& S_{e}=\{x: x e \text { is in } H(e)\}, \\
& e^{S}=\{x: e x \text { is in } H(e)\} .
\end{aligned}
$$

These are subsemigroups of $S$. For instance, if each of $x$ and $y$ is in $S_{e}$ then $x y e=x(e y e)=x e y e$ is in $H(e)$. The next corollary indicates the interaction of $G$ with $S_{l}$ for $l$ in $\operatorname{Im}(L)$.

Corollary 6. The function $\Phi$ defined on $O$ by $\Phi(x)=L(G(x))$ is a $C^{1}$ retraction onto $\operatorname{Im}(L)$. Moreover, there is an open set $U$ containing $\operatorname{Im}(L) H(e)$ so that $\Phi^{-1}(\{l\}) \cap U=S_{l} \cap U$ for each $l$ in $\operatorname{Im}(L)$.

Proof. That $\Phi$ is $C^{1}$ follows because each of $L$ and $G$ is $C^{1}$. If $x$ is in $\operatorname{Im}(L) H(e)$ we have seen that $G(x)=x$, so $G(L(G(x)))=L(G(x))$. Thus we have $L G L G=L G$ since $L L=L$ (Theorem $3($ a)), so $\Phi$ is a retraction.

From Theorem 5 we have that $x$ is in $S_{l}$ if $\Phi(x)=l$. We need to show that if $x$ is in $S_{l}$ and near $\operatorname{Im}(L) H(e)$ then $\Phi(x)=l$.

Suppose $l$ is $\operatorname{in} \operatorname{Im}(L)$. The function which sends $w$ in $S$ to $w l$ is a $C^{1}$ retraction, so, by Theorem 1 , for any $z$ in $H(e)$, the set $\{w: w l=l z\}$ is, near $l z$, a $C^{1}$ submanifold of $S$. The argument for Theorem 5 shows we have the existence of a unique continuous function $K$ from some neighborhood of $\mathrm{lz}$ in this submanifold into $\operatorname{Im}(L) H(e)$ satisfying $x L(K(x))=K(x)$. But, for $x$ in the submanifold, both $x L(x l)=x l$ and $x L(G(x))=G(x)$. Hence there is an open set of $S$ containing $l z$ so that, if $x$ is in the open set and $x l=l z$, then $G(x)=x l$. It follows that $\Phi(x)=l$.

The following theorem and corollary are analogous to Theorem 5 and Corollary 6 and have similar proofs.

Theorem 7. Suppose $(S, *)$ is a differentiable semigroup, $e$ is an idempotent of $S$, and $R$ is as in Theorem 3. There is an open set $O^{\prime}$ containing $H(e) \operatorname{Im}(R)$ so that there is a unique continuous function $J$ from $O^{\prime}$ onto $H(e) \operatorname{Im}(R)$ satisfying $R(J(x)) x=J(x)$. For each $x$ in $\operatorname{dom}(J)$ we have $J(x)$ is in $H(R(J(x)))$. The function $J$ is a $C^{1}$ retraction. 
Corollary 8. The function $\theta$ defined on $O^{\prime}$ by $\theta(x)=R(J(x))$ is a $C^{1}$ retraction. Moreover, there is an open set $V$ containing $H(e) \operatorname{Im}(R)$ so that $\theta^{-1}(\{r\}) \cap V={ }_{r} S \cap V$ for each $r$ in $\operatorname{Im}(R)$.

Using Theorem 1 and Corollary 6 we can now justify the claim that the $S_{l}$ 's fill up a neighborhood of $e$ in $S$ and, near $e$, are all mutually disjoint and locally homeomorphic. Using Theorem 1 and Corollary 8 we obtain the corresponding statement for the ${ }_{r} S$ 's.

\section{CONCLUSION}

Corollaries 6 and 8 provide tools for the beginning of an understanding of the global structure of $S$. They show that an open set of $S$ is contained in the union of a collection of subsemigroups, each member of which is similar in some sense to each other member.

An example of a differentiable semigroup occurs when $S$ is the Banach space of continuous linear operators on a Banach space $Y$ and $*$ is composition. In what follows we refer to this as the linear case.

Many questions remain. Are the semigroups $S_{e}$ and $S_{l}$ isomorphic or locally isomorphic if $l$ is close to $e$ in $\operatorname{Im}(L)$ ? The answer is yes in the linear case. How do these subsemigroups fit together algebraically near $e$ ?

If $S$ has an identity element 1 then by Theorem 3 we know that $H(1)$ is an open subset of $S$ and is a Lie group. For each $e$ in $E(S)$ we know that $S_{e}$ contains 1. Hence, the common part of $S_{e}$ and $H(1)$ is a subgroup $G_{e}$ of $H(1)$. Is this a Lie subgroup of $H(1)$ ? The function which sends $x$ to $x e$ is a homomorphism from $G_{e}$ to $H(e)$. Is it onto? The answer to both of these is yes in the linear case. If it is yes in general then this would restrict the possibilities for $H(e)$ to be a quotient of a subgroup of $H(1)$.

The analogous questions may be asked about the subsemigroups ${ }_{e} S$.

Finally, in the linear case, each idempotent $e$ is connected to $I$ by the one parameter subgroup $f$ defined by $f(t)=\exp (-t)(I-e)+e$. The image of $f$ is a subset of each of $S_{e}$ and ${ }_{e} S$. Is this the case in general for differentiable semigroups with identity element? If so, this would provide a way to identify $E(S)$ from knowledge of $H(1)$.

In the linear case, idempotents correspond to partitions of $Y$ into the direct sum of closed subspaces. The idempotent projects along one subspace onto the other. Theorem 5 can be interpreted in this situation as implying that each $x$ in $\operatorname{dom}(\Phi)$ leaves the image of $\Phi(x)$ invariant. This is related to the result from [1, p. 136] where it is shown that if a Hilbert space operator is within $1 / 12$ of an orthogonal projection it must have a nontrivial hyperinvariant subspace.

In case one $\operatorname{Im}(\Phi(x))$ is one dimensional, all are. It follows in this situation that all members of $\operatorname{dom}(\Phi)$ have a positive eigenvalue and the corresponding eigenvectors are picked out (essentially by $\Phi$ ) in a $C^{1}$ way. This is related to what is referred to in $[4$, p. 587] as a perturbation theorem. 


\section{REFERENCES}

1. B. Beauzamy, Introduction to operator theory and invariant subspaces, North-Holland, Amsterdam, New York, Oxford, and Tokyo, 1988.

2. G. Birkhoff, Analytical groups, Trans. Amer. Math. Soc. 43 (1938), 61-101.

3. J. Dieudonné, Foundations of modern analysis, vol. 1, Academic Press, New York and London, 1960.

4. N. Dunford and J. T. Schwartz, Linear operators Part I: the general theory, Interscience, New York and London, 1958.

5. J. P. Holmes, Differentiable projections and differentiable semigroups, Proc. Amer. Math. Soc. 41 (1972), 391-395.

6. __ Differentiable semigroups, Colloq. Math. 32 (1974), 99-104.

7. __ Rees products in differentiable semigroups, Semigroup Forum 25 (1982), 145-152.

8. __, A rank theorem for infinite dimensional spaces, Proc. Amer. Math. Soc. 50 (1975), 358-364.

Department of Mathematics (FAT), Auburn University, Auburn, Alabama 36849-5310 